\title{
Requirements Engineering for Well-Being, Aging, and Health (REWBAH): An Overview for Practitioners
}

Meira Levy, Lin Liu, Daniel Amyot, Eric Yu, Muneef Alshammari, Malak Baslyman, Elizabeth Bjarnason, Christopher Bull, Carlos Henrique C. Duarte, Eduard C. Groen, Sami Jantunen, Sylwia Kopczyńska, Lysanne Lessard, Ita Richardson, Jens Weber, Ziqi Wei

Well-being, aging, and health (WBAH) are important aspects of life that affect us all. Requirements for WBAH systems have also become a topic of common interest for researchers from different disciplines. This is unsurprising given that health-related expenses often represent about $10 \%$ of a country's Gross Domestic Product (GDP), according to the World Health Organization [1]. At the same time, there is a growing number of software practitioners working on various technology-enabled solutions for WBAH systems. For example, software systems provide personalized and tailored behavioral change programs for decreasing health risk factors. Yet, many systems and services that promote health or well-being fail, in part due to requirements engineering $(R E)$ issues.

We held the first international Requirements Engineering for Well-Being, Aging, and Health (REWBAH) workshop on August 31, 2020, in co-location with the $28^{\text {th }}$ IEEE International Requirements Engineering Conference (RE'20). REWBAH aimed to foster discussions related to requirements about software systems that support healthcare, that promote well-being, that encourage patients and the population in general to live according to healthy lifestyle recommendations, and that address the specific needs of an aging population. When we discussed initiating this event at the end of 2019, we could not imagine that a pandemic would hit us and change the world, and that the workshop itself would be held in a hybrid mode a few months later in Zurich, Switzerland, but mainly online on Discord! Without a doubt, the COVID19 pandemic resulted in many immediate and long-term issues that require special attention from the software engineering community, in particular related to the health and well-being of elderly populations.

The underlying REWBAH theme is in line with the objectives of the American Healthy People 2030 Framework on health promotion and disease prevention, with a vision for "A society in which all people can achieve their full potential for health and well-being across the lifespan" [2]. In the workshop, ten scientific and experience papers (available online [3]) were presented by requirements engineering and health researchers and practitioners from all around the globe. In parallel, and based on their own experiences and on the workshop discussions, the participants were invited to answer and prioritize many questions on a collaborative document, in order to foster ongoing discussions on REWBAH and a related research agenda. Figure 1 shows a word cloud of the participants' answers in that document. The following insights summarize the answers to the subset of questions of particular relevance to software practitioners.

\section{Characteristics of WBAH Applications}

Well-being and health are determined by many factors beyond technological ones, including cultural, habitual, social, and personal factors. Accordingly, requirements elicitation should pay specific attention to soft requirements that are based on such factors but also on an individual's preferences, which are necessary for promoting engagement and continuous use. 
The impact of WBAH applications on health and well-being are hard to measure since such impact takes time and differs among various individuals. However, it is important to measure user's compliance and applications' usage for impact validation. Moreover, assessment frameworks/methods of end-user acceptance and readiness of healthcare organizations must be adapted for pursuing WBAH applications in these organizations.

Another important characteristic is that WBAH applications are always only a fraction of the solution towards well-being and health outcomes. It is important to understand how the applications support and interact with other non-technical WBAH solutions. At the same time, such applications must often integrate with traditional health information systems, leading to security, safety, and privacy concerns. In all cases however, what is measured by the application must assess convergence towards desired outcomes in a valid way.

For WBAH applications to be successful, they must use and be described in an accessible language that the end-users, lawyers, and regulators can understand. There are many examples where the language used is too clinical/technical and misunderstood. WBAH applications are at times invasive and intrusive, collecting and sharing highly sensitive information that again raise privacy, safety, and security concerns.

\section{Top WBAH Challenges}

To the question "What are the top challenges related to Well-Being, Aging, and Health in general?", most of the participants agreed that concepts related to WBAH first need to be defined better as there are different perceptions regarding various domains. For example, health is usually measured more objectively than well-being, healthy behaviors are not necessarily pleasurable, and people may have different views on what "quality of life" represents. Different domains of WBAH should be further analyzed to understand commonalities and differences. This challenge can certainly benefit from merging knowledge and work from multiple disciplines and domains.

Other top challenges for WBAH systems that were identified include, in order of frequencies among the participants' answers:

- Balancing user needs with technical limitations and costs for adapting software.

- Properly considering the high number of adjacent domains, ranging from medicine (to ensure medical validity), design (such as color use for color blindness and visual impairment), interaction (such as how elderly or disabled people interact with applications, especially with changing technological competence), and healthcare, to more specialized aspects in the social domain such as culture, addiction, inequalities, and other social factors.

- Communicating with specialized (and busy) domain experts and other stakeholders, and finding suitable trade-offs between their conflicting goals.

- Merging considerable knowledge and work from multiple disciplines related to WBAH.

- Communicating WBAH user needs and domain knowledge to software engineers.

- Considering ethical and legal aspects in this increasingly regulated domain.

- Developing multi-stakeholder platforms across boundaries of institutes and private homes as we are moving from point-of-care towards continuum-of-care systems.

\section{Top REWBAH Research Challenges}

There are many important research challenges, also relevant to practitioners and entrepreneurs, that were observed in the REWBAH workshop's papers, including:

- Modeling human activities in health-related services (both in digital and physical spaces), incorporating communication, cognition, and emotional theories $[4,5]$; 
- Evaluating and adopting evidence-based approaches in research and practice for wellbeing, especially in regards to the use of Artificial Intelligence [6,7];

- Developing (Web) services and applications for the aging group and combining qualitative and quantitative methods for realizing system requirements $[8,9]$;

- Supporting systems for health-related decision and error detection [10], and health knowledge transfer [11];

- Developing well-being services in the work environment [12] or for people with intellectual disabilities [13].

All these studies realized the importance of addressing various stakeholders and providing improved interactions between people and systems, particularly in points of care where empathic communication is required, in addition to considering ethics and privacy in health-related systems. This last point was actually identified as the top REWBAH research challenge in the participants' answers.

Additional top research challenges relate to addressing the continuum of care from clinics and hospitals to homes; collaborating with well-being and health domain experts to understand their perceptions and together identify relevant problems worth investigating and solving; considering aging as a journey, in the sense of developing a more holistic view of health and well-being (young people are also aging after all); making RE for WBAH more accessible to and easier to adopt by practitioners in order to improve the likelihood of real and sustainable societal impact; and applying evaluation methods from other domains that measure well-being.

\section{Top REWBAH Research Opportunities}

In terms of top REWBAH research opportunities, the participants highlighted Personalized Requirements Engineering, akin to personalized medicine, as they recognized that "one size fits all" does not apply in WBAH from an RE perspective. This also relates to the emerging trends related to digital twins. Other top opportunities include:

- Defining RE processes more appropriate for WBAH and point out their differences from those existing for e-health in general;

- Educating for WBAH recognition;

- Developing domain-specific modeling languages;

- Adopting and adapting systems-engineering approaches in WBAH to better understand the "big picture";

- Studying interactions between patients and medical devices and how these interactions influence well-being.

\section{Multidisciplinarity}

One question for the participants was related to "How can RE help handle the complexity and the multidisciplinary nature of WBAH?" Among the top answers, it was observed that RE can already help with its existing methods (elicitation, modeling, analysis, etc.), technologies, and tools, to identify relevant stakeholders (patients/users, providers, insurers, governments, etc.), capture and understand their needs and powers, and negotiate conflicting objectives. Diversity and multidisciplinarity are often seen as strengths in RE, more so than in many other academic areas that are often siloed. For example, they can help better consider transparency into the data and decisions leading to identified systems requirements and help avoid groupthink issues.

As discussed previously, there exist opportunities to better optimize current RE methods and tools to the many categories of stakeholders, needs, and constraints specific to WBAH applications. 


\section{REWBAH and COVID-19}

The COVID-19 pandemic challenged the world and revealed the need for data-driven, rapid, and agile RE practices and for coping with problems stemming from social challenges such as the digital divide and the massive and specific difficulties posed to people of different ages. There are still issues on that side, as evidenced by the mitigated success of recent mobile contact-tracing applications across the world [14]. REWBAH can for example identify specific challenges of uncertainty, anxiety, and loneliness from different age perspectives, and help assess how emerging home-office/mixed-office environments influence the well-being and health of people so that new requirements for existing and new products can be elaborated. As the pandemic has accelerated the digital transformation of many large-scale organizations and institutions from different sectors, other major opportunities arise from education to critical infrastructures to justice, and importantly from our perspective, in health. The impact of this pandemic will last for years and REWBAH can help us better cope with its negative effects while enabling humanity to better prepare for the next crisis.

\section{What is Next?}

To conclude, this first Requirements Engineering for Well-Being, Aging, and Health workshop was certainly timely and has shown great potential to create an influential, multidisciplinary, and unique community that studies and extends human-centered RE for developing WBAH systems. During the COVID-19 pandemic, WBAH has also emerged as a key socially-challenging topic. We need to invest time and effort in addressing the many challenges identified above, remembering that this list is far from exhaustive. We must also pursue the opportunities identified here and future ones while developing research and practices that foster understanding of how software and technology can engage and help people in improving their well-being and health along their aging journey. Get ready for our next workshop, and stay well and healthy!

\section{Acknowledgment}

We wholeheartedly thank the RE'20 organizers for providing us with the infrastructure needed to host this successful workshop, in particular the RE'20 co-chairs (Martin Glinz and Samuel Fricker), the workshop co-chairs (Sepideh Ghanavati and Luciano Baresi), the proceedings cochairs (Eric Knauss and Patrick Mäder), the Discord infrastructure guru (Oliver Karras), and our efficient student volunteer (Julian Frattini). We also thank the many authors and other participants for fruitful discussions at this event.

\section{References}

1. "Current health expenditure (\% of GDP)." The World Bank Group. Accessed Jan. 30, 2021. [Online]. Available at https://data.worldbank.org/indicator/SH.XPD.CHEX.GD.ZS

2. "Healthy People 2030 Framework". U.S. Department of Health and Human Services. Accessed Jan. 30, 2021. [Online]. Available https://health.gov/healthypeople/about/healthypeople-2030-framework

3. D. Amyot, M. Levy, L. Liu, and E. Yu, (Eds.), 2020 IEEE First International Workshop on Requirements Engineering for Well-Being, Aging, and Health (REWBAH). IEEE CS, 2020. doi: 10.1109/REWBAH51211.2020.

4. N. N. B. Abdullah et al., "Using Work System Design, User Stories and Emotional Goal Modeling for an mHealth System," in 2020 IEEE First International Workshop on Requirements Engineering for Well-Being, Aging, and Health (REWBAH), 2020, pp. 1-10, doi: 10.1109/REWBAH51211.2020.00007. 
5. M. Levy, "Emotional Requirements for Well-being Applications: The Customer Journey," in 2020 IEEE First International Workshop on Requirements Engineering for Well-Being, Aging, and Health (REWBAH), 2020, pp. 35-40, doi: 10.1109/REWBAH51211.2020.00011.

6. M. Thinyane and L. Goldkind, "A Multi-Aspectual Requirements Analysis for Artificial Intelligence for Well-being," in 2020 IEEE First International Workshop on Requirements Engineering for Well-Being, Aging, and Health (REWBAH), 2020, pp. 11-18, doi: 10.1109/REWBAH51211.2020.00008.

7. S. Jantunen, A. Pesola, P. Janhunen, T. Reijonen, and V. Kuuluvainen, "Developing Wellbeing Service Effectiveness Platform: Some RE Considerations," in 2020 IEEE First International Workshop on Requirements Engineering for Well-Being, Aging, and Health (REWBAH), 2020, pp. 19-24, doi: 10.1109/REWBAH51211.2020.00009.

8. B. Ahmad, I. Richardson, and S. Beecham, "A Multi-method Approach for Requirements Elicitation for the Design and Development of Smartphone Applications for Older Adults," in 2020 IEEE First International Workshop on Requirements Engineering for Well-Being, Aging, and Health (REWBAH), 2020, pp. 25-34, doi: 10.1109/REWBAH51211.2020.00010.

9. Z. Wei, Y. Liu, L. Liu, E. Yu, J. Mylopoulos, and C. K. Chang, "Understanding Requirements for Technology-Supported Healthy Aging," in 2020 IEEE First International Workshop on Requirements Engineering for Well-Being, Aging, and Health (REWBAH), 2020, pp. 47-56, doi: 10.1109/REWBAH51211.2020.00017.

10. W. Hutchinson, S. Helal, and C. Bull, "GP Benchmark: Engineering a Crowd-Sourcing Platform for Real-Time Understanding of Personality and Cognitive Biases in Clinical Error," in 2020 IEEE First International Workshop on Requirements Engineering for Well-Being, Aging, and Health (REWBAH), 2020, pp. 41-46, doi: 10.1109/REWBAH51211.2020.00012.

11. I. Davies, J. H. Weber and M. Price, "Translating Advances in Medical Knowledge to Software Requirements: The Lead User Requirements Engineering Method - LURE," in 2020 IEEE First International Workshop on Requirements Engineering for Well-Being, Aging, and Health (REWBAH), 2020, pp. 77-86, doi: 10.1109/REWBAH51211.2020.00015.

12. E. Håkansson and $E$. Bjarnason, "Including Human Factors and Ergonomics in Requirements Engineering for Digital Work Environments," in 2020 IEEE First International Workshop on Requirements Engineering for Well-Being, Aging, and Health (REWBAH), 2020, pp. 57-66, doi: 10.1109/REWBAH51211.2020.00013.

13. M. Alshammari, O. Doody and I. Richardson, "Software Engineering Issues: An exploratory study into the development of Health Information Systems for people with Mild Intellectual and Developmental Disability," in 2020 IEEE First International Workshop on Requirements Engineering for Well-Being, Aging, and Health (REWBAH), 2020, pp. 67-76, doi: 10.1109/REWBAH51211.2020.00014.

14. M. Bano, D. Zowghi, and C. Arora, "Requirements, Politics, or Individualism: What Drives the Success of COVID-19 Contact-Tracing Apps?," IEEE Soft., vol. 38, no. 1, pp. 7-12, 2021. doi: 10.1109/MS.2020.3029311. 


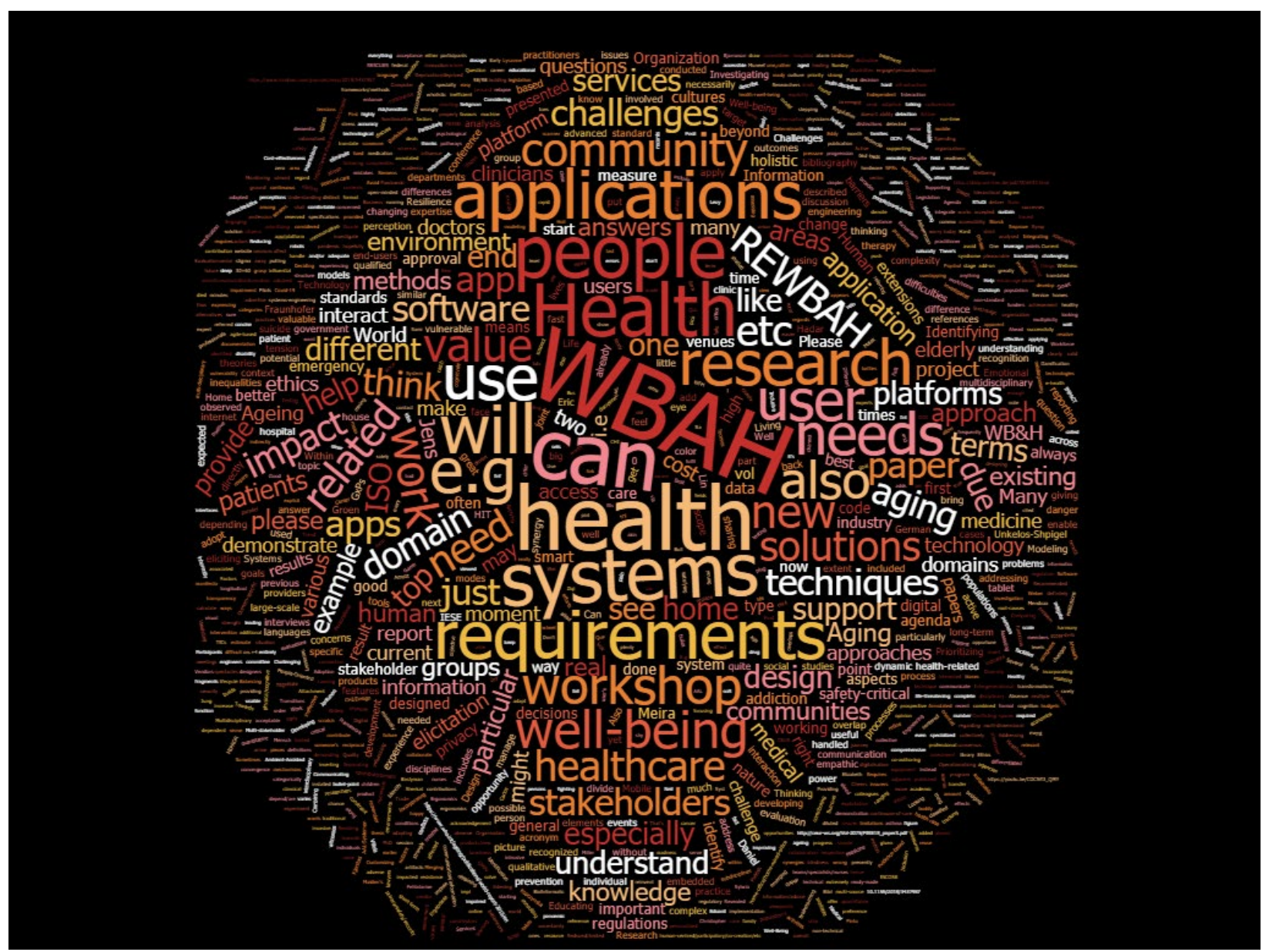

Figure 1: Word cloud based on the answers collected on our collaborative document. 